\title{
Estimation of Claudin 3 in the Sreum of Alopecia Areata Patients and Its Association with Disease Severity
} I.Y.Abdallah ${ }^{1}$, H.E.Abd Elaal ${ }^{1}$, R.A.Khashaba ${ }^{2}$ and D.A.El Salawy ${ }^{1}$

${ }^{1}$ Dermatology and Andrology, Dept., Faculty of Medicine, Benha Univ., Benha, Egypt

${ }^{2}$ Clinical and chemical pathology, Dept., Faculty of Medicine, Benha Univ., Benha, Egypt

E-mail: dr_dodo_89@hotmail.com

\begin{abstract}
Background: Alopecia areata (AA) is a common immune-mediated condition characterized by non-scarring hair loss. The aim of this study was to assess the plasma concentration level of Claudin 3 in patients with alopecia areata and its correlation with the disease severity. Methods: This study included 60 cases of alopecia areata of different types, and 20 apparently healthy volunteers as controls with matched age and sex. All groups were subjected to complete history taking, and through clinincal examination. Severity of AA lesions was assessed using
\end{abstract}

Keywords; Claudin 3, Alopecia, Areata, Severity

\section{Introduction}

As a result of an immune-mediated illness known as Alopecia areata (AA), many people suffer from non-scarring hair loss. An estimated $1.7 \%$ to 2.1 percent of patients in their lifetime will have AA; the frequency increases with age, although there is no substantial difference in the incidence between men and females [1].

Patch AA (PA), alopecia totalis (AT), and alopecia universalis (AU) are all clinical subtypes of AA [2].

Mathematical analysis of hair loss and hair regeneration, known as the Severity of Alopecia Tool (SALT), is routinely used to assess AA severity in the clinic. Each of the four primary regions of scalp hair loss is examined separately (each of the sides, top, and top of the back, representing 18 percent , 18 percent , 40 percent , and 24 percent of the total scalp surface area, respectively). These subscores are combined to arrive at a total percentage of hair loss, known as the SALT score, based on their surface area [3]. Patients with at least $50 \%$ scalp hair loss, including those with $\mathrm{AT}$ and $\mathrm{AU}$, are considered to have severe or widespread AA, according to the American Academy of Dermatology. Molecular tissue scores for treatment response, including the evaluation of baseline and post-treatment levels of hair keratins and inflammatory markers, have also been suggested recently [4].

Intestinal barrier development is promoted by gastrointestinal colonisation, but dysbiosis interferes with this process and increases gut permeability. The immune system is activated when microorganisms, poisons, and metabolites enter the bloodstream. Epithelial cells of the gastrointestinal epithelial barrier form a network of tight connections [5].

Claudins are transmembrane proteins that aid in the establishment of tight junctions by interacting with the actin cytoskeleton. Gut barrier function is impaired when particular claudins are mutated. As a biomarker of intestinal permeability, Claudin 3 in the blood is evaluated [6].

Patients with alopecia areata were included in this research in order to determine the amount of
Claudin 3 in their plasma and its link with disease severity.

\section{Patients and methods}

This case-control study, was conducted on 60 patients suffering from AA (Group A). In addition, 20 apparently healthy individuals of matched age and sex were chosen as a control group (Group B). All patients were selected from the Outpatient Clinic of Dermatology and Andrology Department of Benha University Hospitals.

This study was approved by the local ethic committee on research involving human subjects of Benha Faculty of Medicine.

\section{Inclusion criteria:}

- Patients with localized and diffuse alopecia areata with severity estimation by SALT score [3].

- Patients with newly developed lesions.

\section{Exclusion criteria:}

- Any participant presenting with any other dermatological conditions or any psychiatric disorder.

- History of any systemic diseases e.g. liver diseases, diabetes mellitus, hyperlipidemia or hypertension.

- History of acute gastrointestinal infection during the last 3 months prior to the study.

- Intake of antibiotics, probiotics or prebiotics during the last 3 months.

- Dietary restrictions during the last 3 months.

- History of gastrointestinal surgery during the last 6 months.

- Chronic gastrointestinal disorder (celiac disease, inflammatory bowel diseases, irritable bowel disease, food allergies).

- Pregnancy and breastfeeding.

All groups were subjected to complete history taking, and through clinical examination. Sites, number, morphology and configuration of AA lesions were recorded. Severity of AA lesions was assessed using SALT score. SALT score is a mathematical approach to determine percentage of hair loss in the scalp. 
The scalp was divided into the following 4 areas:

- Vertex: $40 \%(0.4)$ of scalp surface area.

- Right profile of scalp: $18 \%(0.18)$ of scalp surface area.

- Left profile of scalp: $18 \%$ (0.18) of scalp surface area.

- Posterior aspect of scalp: $24 \%(0.24)$ of scalp surface area.

Percentage of hair loss in any of these areas is the percentage hair loss multiplied by percent surface area of the scalp in that area. SALT score is the sum of percentage of hair loss in all the above-mentioned areas as shown in Olsen/Canfield tool [3]. Hair pull test conducted at the periphery of the lesion may be correlated with disease activity. The technique is to do gentle tug at a handful of hair along the edge of an alopecic patch with less strength than would be required to pull out healthy hair. In healthy hair, no hair should fall out or ripped hair would be distributed evenly across the tugged portion of the scalp. In cases of AA, hair will tend to pull out more easily along the edge of the patch [7]. Estimation of serum Claudin 3 levels was done using ELISA technique.

\section{Statistical methods}

SPSS version 25 was used for data administration and statistical analysis. New York, United States (IBM Armonk, New York). Kolmogorov-Smirnov, Shapiro-Wilk, and direct data visualisation approaches were used to determine the normality of quantitative data (for both). Means and standard deviations (or medians and ranges) were used to describe numerical data in accordance with normality testing. Categorical data was presented in the form of percentages and figures. The Mann-
Whitney $\mathrm{U}$ test and the independent t-test were used to compare quantitative data across study groups for normally and non-normally distributed numerical variables. The Chi-square test was used to compare categorical data. It has been determined that claudin can be used to diagnose alopecia. The AUC with a 95 percent confidence interval, the optimal cut-off point, and diagnostic indices were determined. Spearman's correlation was used for the correlations. The MannWhitney $U$ test was used to compare Claudin's performance on a variety of metrics. To predict alopecia, a multivariate logistic regression model was used. The odds ratio and the $95 \%$ confidence interval were derived. "There were no one-sided statistical tests. P-values less than 0.05 were regarded as statistically significant.

\section{Results}

The study was performed on 60 cases of alopecia areata who attended the Outpatient Clinic of the Dermatology \& Andrology Department, Benha University Hospital. The control group consisted of 20 volunteers with matched age and sex. No significant difference was reported between both groups regarding age $(\mathrm{P}$-value $=0.479)$, sex $(\mathrm{P}$-value $=0.538)$, and smoking $(\mathrm{P}$-value $=0.299)$ Table $(1)$

Most patients $(82.0 \%)$ showed sudden onset. About three quarters (72.0\%) showed progressive course. The median disease duration was one month and ranged from 0.3 to 6 months. More than onethird $(40.0 \%)$ showed recurrence. About two-thirds of the patients reported relation to psychological stress, and about one-third reported a history of associated disease. Only $8 \%$ showed family history. Table (2).

Table (1) General characteristics in both groups

\begin{tabular}{|c|c|c|c|c|}
\hline & & Cases $(n=60)$ & Controls $(n=20)$ & P-value \\
\hline Age (Years) & Mean \pm SD & $27 \pm 11$ & $25 \pm 9$ & 0.479 \\
\hline Sex & Males $\quad \mathrm{n}(\%)$ & $45(70.0)$ & $19(63.3)$ & 0.538 \\
\hline & Females $\mathrm{n}(\%)$ & $15(30.0)$ & $11(36.7)$ & \\
\hline Smoking & $\mathrm{n}(\%)$ & $19(38.0)$ & $8(26.7)$ & 0.299 \\
\hline
\end{tabular}

Independent t-test was used for age. Chi-square test was used for sex.

Table (2) Claudin level according to different study parameters

\begin{tabular}{llcc}
\hline & & Claudin $(\mathbf{n g} / \mathbf{m l})$ & P-value \\
\hline Sex & Males & $4.89(4.49-33.7)$ & 0.172 \\
Smoking & Females & $4.32(4-21.9)$ & \\
& Yes & $4.64(4.49-33.7)$ & 0.873 \\
Onset & No & $4.73(4-21.9)$ & \\
& Gradual & $5.76(5-9.18)$ & 0.034 \\
Course & Sudden & $4.76(4.49-33.7)$ & \\
& Stationary & $4.74(4-16.7)$ & 0.582 \\
Recurrence & Progressive & $4.69(4.49-33.7)$ & \\
& Yes & $4.59(4.49-33.7)$ & 0.628 \\
Psychological stress & Yo & $4.81(4.42-21.9)$ & \\
\multirow{4}{*}{ Associated disease } & No & $4.77(4.49-33.7)$ & \\
& Yes & $4.73(4.21-21.9)$ & 0.801 \\
Family history & No & $5.62(5.21-33.7)$ & \\
& Yes & $4.64(4.49-23.8)$ & 0.295 \\
& No & $3.22(3.1-8.93)$ & \\
& & $5.75(4.49-33.7)$ & 0.123 \\
\hline
\end{tabular}




\section{Discussion}

Disturbances in intestinal barrier have been reported in depression. Claudins are transmembrane proteins which participate in the formation of tight junctions by binding to the actin cytoskeleton. Knockout of specific claudins leads to a loss of gut barrier function. Claudin-3 present in the blood is considered as a useful biomarker of intestinal permeability [8]. Changes in microbiota have potent effects on gut permeability [9]. To the best of our knowledge, this is the first study assessing plasma concentration of claudin-3 in patients with alopecia areata. The data presented in this study suggest a possible relationship between alopecia areata, microbiome, gut barrier and immune system.

We assume that changes in the gut microbiota in patients with autoimmune disease lead to intestinal barrier damage [10], and modulate the immune response of the gut. An impairment of the structure and function of the intestinal barrier, intensified by the local inflammation, increases translocation of antigens, bacteria and their toxic products into the systemic circulation [11]. The mechanism contributes to the development of systemic inflammatory response which, in turn, intensifies damage of gut barrier. Our data suggest that breaking proposed vicious circle can improve intestinal barrier function.

Intestinal permeability may be an important target for treatment strategies in alopecia areata. The damage of the intestinal barrier with subsequent translocation of bacterial metabolites into the blood modulates the immune response and influences function of other organs such as skin. This is the basis for creating a concept of a "gut-skin axis" [12].

Sikora and his colleagues measured claudin- 3 in psoriasis, as auto immune skin disease, showed that intestinal permeability, as evaluated by plasma concentration of claudin-3, is increased in patients with psoriasis. Increased gut permeability is associated with high disease activity and smoking. These findings emphasize the importance of intestinal barrier in the pathophysiology of psoriasis. Understanding the interplay between microbiota, gut barrier and inflammation may contribute to the development of new methods of preventing onset or exacerbations of psoriasis [8].

Watson and his colleagues showed claudin-3 was not expressed fully in psoriatic skin, regardless of disease activity. To assess whether the modulation of claudin-3 in psoriasis is a consequence of inflammation, they have studied, in vitro and in vivo, the influence of exogenous cytokines and an experimental inductor of cytokine expression, LPS, on claudin protein expression. Surprisingly, TNF $\alpha$ a major cytokine associated with disease pathology could not modulate claudin expression in their systems. In vitro, they were concerned that this may be due to deficient $\mathrm{TNF} \alpha$ receptor expression; however, Western blot analysis revealed that their cell model expressed both CD120a (TNF-R1) and
CD120b (TNF-R2). In vivo, it is possible that, although signs of inflammation were observed clinically in the volunteers (erythema and edema), their $2 \mathrm{~h}$ biopsy time point was too short to identify changes in claudins. However, work by their group have shown that the TJ is not a static structure and that application of exogenous cytokines and toxins to enterocytes in vitro strip out claudin isoforms from the junctional complex within this time period. This study identify IL- $1 \beta$ as being capable of modulating claudin expression in vitro and in vivo. The IL-1 superfamily of molecules (IL-1 $\alpha$, IL-1 $\beta$, IL-1ra and IL-18) are produced by KCs and play crucial roles in epidermal function. IL-1 $\beta$ is produced also by Langerhans cells and is involved in their migration; it initiates production of other potent pro-inflammatory cytokines, eg IL-8 31, influences adhesion molecule expression in dermal microvasculature, and the IL-1 family expression profile is known to be modified as KCs differentiate. IL-1 $\beta$ has been shown to be expressed at higher levels in psoriatic epidermis and it is therefore likely that this increase can explain their observations on the distribution of claudin isoforms in the involved plaque. Uninvolved, clinically normal skin in patients with psoriasis also shows a change in distribution of claudin- 3 , which can be mimicked in vitro by the addition of IL- $1 \beta$, an observation that implies that even the uninvolved skin is in a pro-inflammatory state [13].

A pathomechanism for the association between disruption of gut integrity reflected by the increase in claudin-3 and AA severity remains unknown. We compared our results with a study that measured claudin-3 in psoriasis as an auto-immune skin disease. The novel and most important finding of Sikora and his colleagues study was a significant, positive correlation of claudin-3 concentration with clinical (Psoriasis Area Severity Index) and laboratory (neutrophil-to-lymphocyte ratio) parameters of disease severity [8]. Also PASI correlated with claudin-3 levels in other recent study [14].

\section{Conclusion}

The serum level of Claudin-3 is significantly higher in patient with alopecia areata compared to controls. It can be used as a predictor for alopecia areata severity.

\section{Source of funding:}

This research did not recive any specific grant from funding agencies in the public,commercial,or not -for-profit sectors.

Author contribution:

Authors contributed equally in the study.

\section{Conflicts of interest:}

No conflicts of interest. 


\section{References}

[1] ACV.Fricke, M.Miteva. Epidemiology and burden of alopecia areata: a systematic review. Clin Cosmet Investig Dermatol.vol.8,pp.397, 2015.

[2] P.Suchonwanit, C.Kositkuljorn, C.Pomsoong Alopecia Areata: An Autoimmune Disease of Multiple Players. ImmunoTargets Ther.vol.10,pp.299,2021.

[3] E.A.Olsen, M.K.Hordinsky, V.H.Price, J.L.Roberts, J.Shapiro, D.Canfield, Alopecia areata investigational assessment guidelinesPart II. J Am Acad Dermatol.vol.51(3),pp.440-7,2004.

[4] Y.Renert-Yuval, E.Guttman-Yassky, The changing landscape of alopecia areata: the therapeutic paradigm. Adv Ther.vol.34(7),pp.1594-609,2017.

[5] J.R.Kelly, P.J.Kennedy, J.F.Cryan, T.G.Dinan, G.Clarke, NP. Hyland, Breaking down the barriers: the gut microbiome, intestinal permeability and stress-related psychiatric disorders. Front Cell Neurosci.vol.9,pp.392,2015.

[6] C.Barmeyer, M.Fromm, J.D.Schulzke, Active and passive involvement of claudins in the pathophysiology of intestinal inflammatory diseases. Pflügers Arch J Physiol.vol.469(1),pp.15-26,2017.

[7] L.Xu, K.X.Liu, M.M.Senna, A practical approach to the diagnosis and management of hair loss in children and adolescents. Front Med.vol.4,pp.112,2017.

[8] M.Sikora, M.Chrabąszcz, A.Waśkiel-Burnat, A.Rakowska, M.Olszewska, L.Rudnicka, Claudin-3-a new intestinal integrity marker in patients with psoriasis: association with disease severity. J Eur Acad Dermatology Venereol.vol.33(10),pp.1907-12,2019.
[9] L.Lin, J.Zhang, Role of intestinal microbiota and metabolites on gut homeostasis and human diseases. BMC Immunol.vol.18(1),pp.125,2017.

[10] Q.Mu, J.Kirby, C.M.Reilly, X.M.Luo, Leaky gut as a danger signal for autoimmune diseases. Front Immunol.vol.8,pp.598,2017.

[11]M.G.Rooks, W.S.Garrett, Gut microbiota, metabolites and host immunity. Nat Rev Immunol.vol.16(6),pp.341-52,2016.

[12] K.Migacz-Gruszka, W.Branicki, A.Obtulowicz, M.Pirowska, K.Gruszka, A.Wojas-Pelc, What's new in the pathophysiology of alopecia areata? the possible contribution of skin and gut microbiome in the pathogenesis of alopeciaBig opportunities, big challenges, and novel perspectives. Int Trichology.vol.11(5),pp.185,2019.

[13] R.E.B.Watson, R.Poddar, J.M.Walker, I.McGuill, L.M.Hoare, Griffiths CEM, et al. Altered claudin expression is a feature of chronic plaque psoriasis. J Pathol A J Pathol Soc Gt Britain Irel.vol.212(4),pp.450-8,2007.

[14] A.A.E.Ahmed, E.Dakrory, F.Faisal, S.R.A.Q.Hendawy, NM.Shaaban, Assessment of Serum Level of Claudin-3 and Its Association with Disease Severity in Patients with Psoriasis Vulgaris. Egypt J Hosp Med.vol.83(1),pp.1155-61,2021. 